4. Esteban A, Ferguson ND, Meade MO, Frutos-Vivar F, Apezteguia C, Brochard L et al (2008) Evolution of mechanical ventilation in response to clinical research. Am J Respir Crit Care Med 177:170-177

5. Franz D, Bunzemeier $\mathrm{H}$, Roeder $\mathrm{N}$, Reinecke H (2010) Krankenhausfinanzierung unter DRGBedingungen. Med Klin 105:13-19

6. Geissler A, Scheller-Kreinsen D, Busse R (2011) Germany: Understanding G-DRGs. In: Busse R, Geissler A, Quentin W, Wiley M (Hrsg) Diagnosisrelated groups in Europe: moving towards transparency, efficiency and quality in hospitals. Open University Press, WHO Regional Office for Europe, Maidenhead, S243-271

7. Geissler A, Scheller-Kreinsen D, Quentin W, Busse R (2012) DRG-Systeme in Europa: Anreize, Ziele und Unterschiede in zwölf Ländern. Bundesgesundheitsbl Gesundheitsforsch Gesundheitsschutz 55:633-642

8. Institut für das Entgeltsystem im Krankenhaus (2012) G-DRG, German diagnosis related groups. Definitions-Handbuch. http://g-drg.de/ cms/Archiv/DRG_Systemjahr_2012_Datenjahr_ 2010\#sm3.Zugegriffen:20.Aug. 2015

9. Institut für das Entgeltsystem im Krankenhaus (2014)Deutsche Kodierrichtlinien. Allgemeine und spezielle Kodierrichtlinien für die Verschlüsselung von Krankheiten und Prozeduren. http://g-drg.de/ cms/Archiv/DRG_Systemjahr_2014_Datenjahr 2012\#sm5.Zugegriffen:20.Aug. 2015

10. Moran JL, Solomon PJ (2012) Mortality and intensive care volume in ventilated patients from 1995 to 2009 in the Australian and New Zealand binational adult patient intensive care database. Crit Care Med 40:800-812

11. Nielson C, Wingett D (2004) Intensive care and invasive ventilation in the elderly patient, implications of chronic lung disease and comorbidities. Chron Respir Dis 1:43-54

12. Rau F, Roeder N, Hensen P (2009) Zum Stand der deutschen DRG-Einführung: Erkenntnisse, Erfahrungen und Meinungen. In: Rau F, Roeder $\mathrm{N}$, Hensen $\mathrm{P}$ (Hrsg) Auswirkungen der DRGEinführung in Deutschland. Standortbestimmung und Perspektiven. Kohlhammer, Stuttgart, S9-22

13. Schreyögg J, Bäuml M, Krämer J, Dette T, Busse R, Geissler A (2014) Endbericht zum Forschungsauftrag gem. § 17 b Abs. 9 KHG. http://www.gdrg.de/cms/Begleitforschung_gem._17b_Abs. 8_KHG/Forschungsauftrag_gem._17b_Abs._9_ KHG. Zugegriffen:20. Aug. 2015

14. Storre JH, Schönhofer B (2008) Noninvasive mechanical ventilation in chronic respiratory failure: ventilators and interfaces. In: Muir J, Ambrosino N, Simonds AK (Hrsg) Noninvasive Ventilation (European Respiratoy Monograph). European Respiratory Society Journals Ltd, Sheffield, S319-337

15. Tuschen KH (2012) Entstehung, Darstellung und Bewertung des DRG-Systems sowie Perspektiven der Weiterentwicklung aus bundesweiter Sicht. In Baum G (Hrsg) Krankenhausentgeltsystem. Deutsche Krankenhaus Verlagsgesellschaft, Düsseldorf, S3-30

16. Zilberberg MD, de Wit M, Shorr AF (2012) Accuracy of previous estimates for adult prolonged acute mechanical ventilation volume in 2020. Crit Care Med 40:18-20

\title{
Erratum
}

Anaesthesist 2016 $65: 672$

DOI 10.1007/s00101-016-0210-3

Online publiziert: 9 . August 2016

๑) Springer-Verlag Berlin Heidelberg 2016

CrossMark

M. T. Zacher ${ }^{1}$ A. M. Högele' - M. Hanschen ${ }^{1}$ - F. von Matthey ${ }^{1}$ A.-K. Beer² F. Gebhardt ${ }^{2}$ P. Biberthaler ${ }^{1} \cdot$ K.-G. Kanz ${ }^{1}$

'Klinik und Poliklinik für Unfallchirurgie, Klinikum rechts der Isar, Technische Universität München, München, Deutschland

${ }^{2}$ Institut für Mikrobiologie, Immunologie und Hygiene, Klinikum rechts der Isar, Technische Universität München, München, Deutschland

\section{Erratum zu: Grundlegende Techniken des Wundverschlusses in der Notaufnahme}

\author{
Erratum zu: \\ Anaesthesist 2016 • 65:303-324 \\ DOI 10.1007/s00101-016-0170-7
}

In Tab. 2 des Beitrages liegt ein Fehler vor: Nicht bei Lidocain, sondern bei Prilocain kann in seltenen Fällen $(\geq 1 / 10.000$ bis $<1 / 1000)$ eine Methämoglobinämie als spezifische Nebenwirkung auftreten. Zudem ist diese Substanz bei angeborener oder erworbener Methämoglobinämie mit einer entsprechenden Gegenanzeige versehen.

Wir bitten, diesen Fehler zu entschuldigen.

\section{Korrespondenzadresse}

\section{T. Zacher}

Klinik und Poliklinik für Unfallchirurgie, Klinikum rechts der Isar, Technische Universität München

Ismaninger Str. 22, 81675 München,

Deutschland

martina.zacher@tum.de
Die Online-Version des Originalbeitrages finden Sie unter doi:10.1007/s00101-016-0170-7 